\title{
Derechos sociales: difíciles de construir, fáciles de destruir
}

\author{
Social rights: difficult to construct, easy to \\ destroy
}

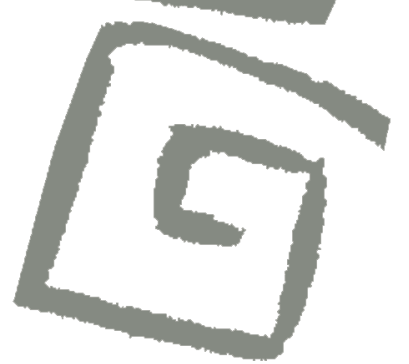

Sonia Fleury

'Socióloga. Doctora en Ciencias Políticas. Investigadora principal, Centro de Estudos Estratégicos, Fundação Oswaldo Cruz Rio de Janeiro, Brasil. $\bowtie$ iD
RESUMEN El objetivo de este trabajo es reflexionar, desde el caso de Brasil, sobre las tensiones actuales entre el sistema de protección social -que en los últimos 30 años se organizó como derechos de la ciudadanía y como parte de la construcción de la democracia en el país- y el proceso de desmantelamiento en el período actual por un régimen populista autoritario. Desde la perspectiva teórica presente en este trabajo, se indaga sobre las características estructurales y los factores de la coyuntura que nos permiten explicar por qué ha sido tan difícil la construcción de la arquitectura legal e institucional de los derechos sociales y, al revés, por qué resulta tan fácil en la actualidad destrozarla en la práctica.

PALABRAS ClAVES Protección Social; Ciudadanía; Política Pública; Brasil.

ABSTRACT The purpose of this article is to reflect on contemporary tensions between the social protection system in Brazil - which in the past 30 years has come to be organized as a social right and has been part of the construction of democracy in the country - and the current process of its dismantling under an authoritarian populist regime. From the theoretical perspective adopted in this article, structural characteristics and circumstantial factors are examined in order to explain the difficulties that have been faced in constructing a legal and institutional architecture for social rights, and on the other hand, why at present it is so easy to destroy it in practice.

KEY WORDS Social Protection; Citizenship; Public Policy; Brazil. 


\section{INTRODUCCIÓN}

Este artículo se estructura en tres secciones. En la primera se discute la inadecuación de la teoría del welfare state -desarrollada a partir de estudios europeos- para su aplicación en contextos distintos. Luego, abordamos los estudios sobre el desarrollo de la protección social en América Latina. Y, por último, analizamos el montaje del Estado de bienestar en Brasil y su rápido proceso de desmantelamiento en los años recientes, buscando identificar los obstáculos en su trayectoria, los avances y las debilidades que permiten un rápido proceso de deterioro.

\section{La teoría eurocentrada del welfare state}

La teoría sobre la emergencia de los Estados de bienestar social o welfare state está netamente dirigida a la realidad europea de fines del siglo XIX y comienzos del siglo XX, e identifica a algunos procesos sociales como responsables de la emergencia y, a otros, como responsables de las características que asume la protección social en cada contexto nacional.

El welfare state es considerado una articulación peculiar de conflictos distributivos, particularmente, el conflicto entre la lógica de mercado y los principios de asignación política, centrados en los arreglos institucionales de las políticas sociales en cada sociedad. Se considera que el punto de partida es el proceso de industrialización ${ }^{(1)}$ y el surgimiento del tema de la inseguridad. Se considera que la ruptura de los lazos comunitarios tradicionales de solidaridad y su reemplazo por la organización e identidad de clase, así como el desarrollo de la función pública y las estructuras administrativas estatales, están en las raíces del desarrollo del welfare state. La conclusión de Wilensky ${ }^{(2)}$ es que el welfare state es la tendencia estructural más persistente en el desarrollo de las sociedades, a pesar de los regímenes ideológicos y políticos, asociados con el desarrollo económico y el proceso de modernización social.
A diferencia de esta perspectiva no histórica, otros autores han resaltado los determinantes de los sistemas universales de protección social, ya que una misma demanda de protección social recibió diferentes respuestas según el entorno institucional, político y económico previo. Flora y $\mathrm{Alber}^{(3)}$ identifican el surgimiento del modelo universal de protección social como dependiente de la fuerza de una clase trabajadora homogénea en la lucha por los derechos sociales, así como de la capacidad de cada sociedad para institucionalizar este conflicto a través de procedimientos democráticos.

Para Offe ${ }^{(4)}$, el welfare state y el sistema de partidos políticos competitivos son las principales características para promover la convivencia del capitalismo y la democracia en su ciclo virtuoso, ya que transforman la competencia política en mercancías y la economía privada en una cuestión política. El desarrollo de la protección social y la expansión de la ciudadanía fueron vistos como reacciones a las tendencias disruptivas del capitalismo con la expansión de la ciudadanía en una sociedad de masas ${ }^{(5,6)}$.

Se trata de un nuevo acuerdo entre el Estado, el mercado y los ciudadanos, en el cual el Estado asume un rol de regulador de las relaciones productivas $y$, por medio de imposiciones tributarias, alcanza a establecer las bases materiales de la redistribución del acuerdo con el conjunto de derechos atribuidos a la comunidad de ciudadanos. En este sentido, los conflictos se trasladan de la esfera de la producción a la de la reproducción y se produce algún grado de desmercantilización de la reproducción ${ }^{(7)}$. El welfare state fue responsable de forjar nuevos lazos en sociedades complejas y también de crear un nuevo patrón institucional de resolución redistributiva de conflictos, sentando las bases para la cohesión e integración social.

La pérdida de centralidad de la "sociedad salarial" ${ }^{\prime(8)}$ con la incorporación de las innovaciones tecnológicas que transformaron los procesos productivos y generaron más informalidad y aumento de la desigualdad, la hegemonía del pensamiento neoliberal y de las políticas de austeridad, son factores que 
han impactado fuertemente en las economías desarrolladas y en sus mecanismos de protección social. La transformación de los Estados, de recaudadores a deudores ${ }^{(9)}$, redujo las condiciones fiscales para el ejercicio de las políticas redistributivas, desilusionando a los ciudadanos con las limitaciones de la democracia.

Pierson $^{(10)}$ hace hincapié sobre la diferencia entre los factores que la teoría identifica como explicativos de la emergencia y la expansión del welfare state y aquellos que pueden explicar el grado de resiliencia a las medidas de austeridad. En las economías afluentes se estima que la mitad de la población es, de manera directa o indirecta, beneficiaria de los diferentes programas de protección social. O sea, la expansión de la cobertura y del rol de beneficios se corresponde al aumento del número de involucrados en la protección social que utilizan el voto para evitar la pérdida de sus beneficios. Por otra parte, la trayectoria institucional de los sistemas de protección también funciona como un factor que puede o no provocar mayor resiliencia a cambios. Distingue tres tipos de medidas utilizadas en la contención de costos: remercantilización, reducción, recalibración. En conclusión, afirma:

La segunda observación es que, si bien las agendas de reforma varían sustancialmente entre los tipos de régimen, todas dan prioridad a la contención de costos. Este énfasis compartido refleja el inicio de la austeridad permanente. Los Estados de bienestar no se están desmantelando. Los esfuerzos para lograr la recalibración pueden generar innovaciones interesantes e incluso ampliar la provisión social. Sin embargo, en todas partes, estos ajustes se producen en un contexto en el que el control del gasto público no solo es una consideración central, sino dominante. Las estructuras centrales de la mayoría de los Estados de bienestar no están en peligro. No obstante, el clima contemporáneo sigue siendo duro para los esfuerzos por mejorar la provisión social para los más vulnerables o para abordar los riesgos recientemente reconocidos. [Traducción del original: The second observation is that while reform agendas vary quite substantially across regime types, all of them place a priority on cost-containment. This shared emphasis reflects the onset of permanent austerity. Welfare states are not being dismantled. Efforts to achieve recalibration can generate interesting innovations and even extensions of social provision. Yet everywhere, such adjustments occur in a context where the control of public expenditure is a central if not dominant consideration. The core structures of most welfare states are not in jeopardy. Nonetheless, the contemporary climate remains a harsh one for efforts to improve social provision for the vulnerable, or to address newly-recognized risks]. ${ }^{(10)}$

\section{Protección social en economías emergentes y democracias rezagadas}

Uno puede preguntarse qué pasa con la protección social cuando esas condiciones no se reproducen como en América Latina: la industrialización no ha generado una clase más homogénea de trabajadores; la economía no produce condiciones de desarrollo sostenibles; los acuerdos interclases, que permiten un pacto socialdemócrata no son viables; los Estados tienen bajas capacidades regulatorias, recaudatorias e institucionales para asegurar los derechos sociales, y los actores internacionales tiene alto peso en las decisiones públicas. Ese es el caso de las sociedades de economías dependientes en democracias rezagadas que intentan construir un sistema de protección social como respuesta a demandas sociales policlasistas.

A diferencia de otros países donde la política pública de protección social es más reciente, los países del Cono Sur en América Latina tienen una larga historia iniciada en el viraje del siglo XIX al siglo XX, en el campo de las políticas sociales. Mesa Lago ${ }^{(11)}$ identificó que se pueden diferenciar tres grupos de países en la región a partir del momento en el que se inicia la protección social: pioneros, 
intermedios y rezagados. Pero en todos ellos se ha establecido un modelo de seguro para los trabajadores del mercado formal, en economías con elevado grado de informalidad laboral. Aun en los países pioneros, en los cuales la protección social estuvo asociada al proceso de industrialización, la clase obrera siguió siendo predominantemente informal, o sea, sin estar cubierta por el seguro social. Entre los que estaban cubiertos, la característica más relevante fue la estratificación de la protección de acuerdo con la capacidad de organización de los grupos de presión según su posición estratégica en el modelo económico. Aunque con el proceso de maduración del sistema, se expandió la cobertura, la característica de estratificación continuó siendo significativa. Estudios más recientes, como el de Filgueiras ${ }^{(12)}$, han tratado de añadir a la característica de estratificación el grado de exclusión de la población, encontrando los tipos "universalismo estratificado", "sistemas duales", y los predominantes "excluyentes".

Steffen y Côrtes ${ }^{(13)}$ clasifican los estudios sobre la protección social en América Latina en tres ejes. El primero, en el cual incluyen los estudios de Mesa Lago y de Filgueiras, se caracteriza por tener como foco la cobertura y el gasto social. El segundo, incluye los estudios en los cuales el foco está en la mezcla (welfare mix) de organizaciones públicas y privadas en la provisión de la protección social, con énfasis en el rol de las familias. Los trabajos desarrollados por Martínez-Franzoni ${ }^{(14)}$ son la mejor expresión del esfuerzo por rescatar la importancia del trabajo femenino no pago en la reproducción social. Desde la concepción de Esping-Andersen, que entiende los tipos de instituciones de protección social como el arreglo entre Estado, mercado y hogares, la autora identifica tres tipos de regímenes de protección en la región, dos de ellos centrados en el Estado y un tercero en la familia: Estado-focalizado, Estado-estratificado e informal-familiar. El tercer eje incluye los estudios focalizados en la provisión estatal, que construyen índices para analizar las capacidades estatales, utilizando diferentes indicadores y modelos complejos. Segura-Ubiergo ${ }^{(15)}$ es un buen ejemplo con la proposición de un "welfare effort index" por medio del cual clasifica los países en niveles altos y bajos de esfuerzos en la provisión social.

En resumen, se realizó todo un esfuerzo por desarrollar tipologías que se sucedieron en el tiempo, que en algunos casos se superponen, pero en otros toman países distintos y consideran diferentes aspectos. Metodológicamente, difieren de las tipologías elaboradas para el welfare state en los países europeos por el hecho de que no se tratan de tipos ideales deducidos de un análisis histórico social consistente del desarrollo de la protección social, siendo más bien una clasificación a partir de categorías nominales o indicadores numéricos. En su pertinente comentario acerca de los estudios sobre la protección social en la región, Gómez ${ }^{(16)}$ termina por concluir que explican bien el "cómo" son los sistemas de protección en la región, pero no "por qué" son así. En su análisis, identifica una trayectoria de estudios que siguen la dinámica de las propias políticas, inicialmente centrados en las tipologías de los seguros sociales, pasando a las políticas que favorecieron la privatización y el crecimiento del sector privado en la provisión de servicios, retornando al Estado con las políticas de transferencias condicionadas de ingresos para combatir la pobreza.

Algunas cuestiones teóricas ameritan ser consideradas para la comprensión de la protección social en América Latina: la naturaleza del Estado, el régimen político y la categoría apropiada para comprender las instituciones de la protección social.

Al analizar la protección social en América Latina buscamos comprender las políticas públicas como una mediación entre Estado y sociedad, permitiendo así entender el espacio que esa política ocupa en el interior del aparato estatal y su rol en la reproducción social. Una política social tiene su emergencia a partir de la cuestión social, puesto que considero que el surgimiento de una cuestión social presupone una nueva correlación de fuerzas y la creación de un tejido social alrededor de tal problemática, con capacidad para insertarla en la agenda pública. Sin embargo, la puesta en marcha no asegura el 
control de su encuadre ni su sustentabilidad. Se trata de analizar la política como parte de la lucha por la hegemonía y ampliación del Estado $^{(17)}$, lo que presupone la constitución de sujetos políticos, cuya relación de fuerzas impone que el Estado, restringido al núcleo coercitivo, se amplíe con medidas que atiendan las demandas populares y aseguren la legitimidad de la autoridad.

De esa forma se puede revelar tanto la naturaleza del Estado como su dinámica de institucionalización, a través de las estructuras de la protección social. Tratar la política pública a partir de la emergencia de la cuestión social nos permite evitar los modelos tradicionales que consideran al Estado como una caja negra en la cual los actores insertan sus intereses $\mathrm{O}$, al revés, como los que consideran la burocracia estatal como demiurgos que formulan las políticas públicas a partir de una lógica racional en un contexto de escasez, en el que operan elecciones trágicas.

Para comprender la forma del Estado en América Latina, los estudios señalan la dependencia económica y la sujeción a los intereses de reproducción del capital internacional, como los factores responsables de la heterogeneidad económica y el predominio de la esfera política en la unificación del cuerpo social. El predomino de la esfera política es también su fragilidad, capturada por los intereses particularistas, cuya dominación implica el uso recurrente de la violencia contra la expresión de demandas populares.

La construcción del Estado y de la Nación en la región no ha presentado un proceso secuencial y resolutivo de las cuestiones de unificación, integración, participación y distribución. Por el contrario, su presencia simultánea como desafíos acarrean soluciones parciales e inconclusas que resultan en un híbrido institucional, con niveles escalonados de superposición de estructuras de diferentes matrices, que no se superan, pero se amalgaman en procesos de transición pendientes.

Para estudiar la implementación del patrón de seguridad social en América Latina tomé y comparé países pioneros como Uruguay, Chile, Argentina y Brasil, considerando las condiciones de emergencia, de consolidación del modelo de protección, expansión y masificación y de posterior maduración y crisis ${ }^{(18,19)}$. Las particularidades de cada contexto en la relación Estado/sociedad y la correlación de fuerzas entre las principales clases e intereses involucrados en la arena en la cual se definen las políticas de protección social suelen explicar el grado de amplitud de la cobertura y de generosidad de los beneficios. Los grados de heterogeneidad de la clase obrera, fruto del modelo económico de industrialización por sustitución de importaciones y de la urbanización, definen tanto la formalización laboral como la capacidad de los trabajadores en la disputa del excedente convertido en medidas de protección social.

Sin embargo, se evidencia un mismo patrón fragmentado con relación a la cobertura: estratificado en cuanto a las fracciones de los trabajadores en el mercado laboral formal y urbano, y excluyente con relación a los trabajadores informales, rurales y domésticos. Los trabajadores urbanos del mercado formal fueron incorporados, por un lado, como consumidores, ampliando el mercado nacional $y$, por otro, como socios y legitimadores del pacto de poder, por medio de su cooptación e inserción fragmentada, lo que impidió la posibilidad de la unidad de la clase obrera.

El patrón de protección social en formato de seguro social se ha asociado al modelo de desarrollo económico dependiente y restrictivo, y al ejercicio de poder autoritario y excluyente. Las políticas de protección social involucran necesariamente la disputa por el excedente, ya que buscan reducir la apropiación privada de la riqueza producida por medio de políticas públicas distributivas. Pero la transformación de los beneficios en privilegios corporativos impidió la universalización de la ciudadanía y la democratización de las relaciones sociales. La combinación de inclusión fragmentada y estratificada con la exclusión generalizada de otras fracciones menos organizadas y más empobrecidas de trabajadores reprodujo las jerarquías de estatus al interior de la clase trabajadora. Mi conclusión es que se construyeron Estados sin ciudadanos ${ }^{(19)}$.

Alrededor de esa arena se organizaron grupos de interés que, en el caso del Estado 
de bienestar corporativo del periodo desarrollista, incluían a los trabajadores sindicalizados, la burocracia estatal y los partidos políticos. El agotamiento del modelo económico de industrialización sustitutiva de las décadas de 1960 y 1970 encontró a las economías latinoamericanas debilitadas por la inflación y el endeudamiento, con reducción del poder de los Estados nacionales y de la capacidad política de mantener la coalición que sustentaba las políticas sociales. Trabajadores, burocracia y partidos fueron desplazados por gobiernos dictatoriales asociados a grandes corporaciones transnacionales y subordinados a organismos internacionales como el Banco Mundial, el Fondo Monetario Internacional y el Banco Interamericano de Desarrollo, que condicionaron sus préstamos a las reformas de los sistemas de pensiones y la destrucción de la coalición del período del desarrollismo corporativo.

A partir de la década de 1980 se inicia un período de intensas reformas de los sistemas de protección en la región, en los que se pueden identificar modelos paradigmáticos, como el modelo dual en Chile, el modelo universal en Brasil o el modelo plural en Colombia ${ }^{(20)}$. En Chile, en 1980 bajo la dictadura y en medio de una fuerte crisis económica, se implementó un modelo dual, en el que se separa el sistema público para la población y el sistema de seguro privado para los que presentan capacidad contributiva. Se rompe con el modelo de repartición y con el seguro social, cambiando de beneficios definidos a contribuciones definidas acumuladas en una cuenta individual. En otras palabras, se impone un modelo sin solidaridad y que asume la dualidad socioeconómica como fundamento de la política de protección social, con graves consecuencias con relación a la profundización de las desigualdades y transferencia de riesgos exclusivamente a los participantes.

En Brasil, a fines de la década de 1980, en medio del proceso de transición a la democracia y todavía sin reflejos fuertes de la crisis económica, la protección social se inscribe en la Constitución Federal de 1988 CF/88 como parte de los derechos sociales universales a ser garantizados por el Estado. Se implementan sistemas públicos, descentralizados y participativos que involucran los tres niveles de gobierno en salud y asistencia social. Las pensiones no contributivas para trabajadores rurales pasan a tener un valor vinculado al salario mínimo. En la década de 1990, se implementa un modelo universal, ya en una situación de alta inflacionaria y endeudamiento público, que compromete la concretización del proceso de universalización.

En la década de 1990, en Colombia, en un período de crecimiento económico, se propone un modelo plural (pluralismo estructurado), que busca articular los sectores públicos y privados en dinámicas competitivas en lo que se refiere al aseguramiento y a la provisión de los servicios. Buscando superar los graves problemas de inequidad que se plantearon en el caso chileno, se introduce un componente de solidaridad, costeado por un porcentaje de las contribuciones de los sueldos más altos y por un aporte del gobierno. Sin embargo, surgieron otros problemas vinculados a la gestión de la recaudación: las aseguradoras privadas no estaban interesadas en cobrar pues tenían garantizado el pago per cápita; la falta de aportes solidarios del gobierno en período de crisis económica; el desmantelamiento de la salud pública como consecuencia del modelo competitivo y el uso clientelista de los recursos solidarios por parte de las municipalidades.

Comparando los procesos de reforma de la protección social, encontramos que la variable del timing de las reformas, en relación con los macro-fenómenos del régimen político y de la economía, definió los diseños de las reformas ${ }^{(21)}$. En los casos de los modelos paradigmáticos descriptos hubo re-reformas para adecuarse a los nuevos contextos políticos y económicos. Sin embargo, a pesar del aumento de la cobertura, la estratificación persistió y la privatización se profundizó en la región, sea como eje central del modelo, como en Chile, sea en articulación con el sector público, como en Colombia, sea de manera más subrepticia por adentro del sistema público y también como sistema complementario abonado con subsidios estatales, como en Brasil. 
No se puede hablar de un Estado de bienestar consolidado en los países del Cono Sur, ni siquiera en Costa Rica que ha tomado medidas concretas para universalizar la protección social, puesto que las políticas de austeridad adoptadas por los gobiernos de los años 2000 en adelante, como parte del ajuste fiscal propugnado por los modelos neoliberales de las agencias internacionales, han reducido los recursos destinados a la protección social y aumentado la privatización. La adopción de políticas de transferencias condicionadas de ingresos se extendió por toda la región como forma de mitigar la pobreza sin gran dispendio de recursos públicos. Se creó un sistema mixto en el cual se incluye la protección social para los trabajadores formales y los programas de combate a la pobreza. El resultado es una paradoja en la cual encontramos derechos sin beneficios y beneficios sin derechos de ciudadanía ${ }^{(22)}$, aumentando la judicialización.

Con el intento de englobar de manera más amplia las diferentes formas de protección social, en especial para contemplar países pobres o en desarrollo, autores como Gough y Wood $^{(23)}$ encuentran que el concepto de Estado de bienestar no se aplica con propiedad a países en desarrollo, donde las personas viven en situaciones de inseguridad y es la comunidad o sus familiares quienes cubren sus necesidades. Proponen el concepto de "regímenes de bienestar", definidos como:

\begin{abstract}
El régimen de bienestar es un término más genérico que se refiere a todo el conjunto de acuerdos, políticas y prácticas institucionales que afectan los resultados del bienestar y los efectos de estratificación en diversos contextos sociales y culturales. [Traducción del original: Welfare regime is a more generic term, referring to the entire set of institutional arrangements, policies and practices affecting welfare outcomes and stratification effects in diverse social and cultural contexts]. ${ }^{(23)}$
\end{abstract}

De este modo, se proponen incluir regímenes de inseguridad, en los que las personas no pueden esperar que sus necesidades sean atendidas a través del acceso a los servicios estatales o la participación en el mercado laboral. Si para el Estado de bienestar el criterio de seguridad es la desmercantilización, para el régimen de bienestar el criterio es atender las necesidades con recursos provenientes de las familias y otras relaciones comunitarias.

Nuestra crítica a ambos criterios definidores de tales conceptos se basa en dos argumentos: 1) la intervención estatal, desde los inicios del capitalismo ${ }^{(6)}$ se ha convertido en una estrategia para el fortalecimiento del mercado, y es así como se llega a la privatización, en especial, en los países en los cuales el mercado de servicios sociales tiene como límite la capacidad restringida de consumo de la población; y 2) para igualar diferentes modalidades de previsión social -estatal, mercadológica y familiar- es necesario despojarla de su carácter político jurídico de derecho de ciudadanía, reduciéndola a la atención de necesidades humanas. En ese último caso no se establecen diferencias entre beneficios y derechos, consumidores y ciudadanos.

Al tomar el estatus político de ciudadanía como criterio para calificar a la protección social, estamos postulando que cumple los requisitos del reconocimiento ${ }^{(24)} \mathrm{y}$ de la redistribución ${ }^{(25)}$ por medio de políticas públicas que definen diferentes patrones que involucran un clúster de variables articuladas -ideología, estatus, financiación, organización, etc.- que resultan en distintas formas de ciudadanía: la "ciudanía invertida"(18) cuando predomina la política asistencialista compatible con la ideología liberal; la "ciudadanía regulada"(26) por la inserción el mercado laboral en el modelo corporativo del seguro; la "ciudadanía universal" en las sociedades en las que la social democracia ha instituido la seguridad social ${ }^{(18)}$. A partir de la creación del modelo dual chileno se puede establecer un cuarto tipo ideal en el cual la política pública crea el mercado de previsión social, estableciendo una condición productivista para el acceso a los servicios sociales, y crea el "consumidor social individualizado", reemplazando la condición de ciudadanía.

Más allá de tipologías, tratamos de tipos ideales que suelen convivir en la realidad de 
cada país, aunque una de las formas predomine sobre las demás. La importancia de tomar ese lente político para situar la previsión social es que nos permite entender la constitución, pero también el derrumbe de los sistemas de protección social.

\section{EL DESMANTELAMIENTO DE LA PROTECCIÓN SOCIAL EN BRASIL}

¿Cómo se puede explicar que una de las experiencias más exitosas en la región, de construcción de un sistema de protección social basado en la universalización de la ciudadanía, pueda ser deconstruido en tan poco tiempo? Para responder a esa pregunta es necesario subrayar la trayectoria y las principales características de la protección social en Brasil. Como postulamos anteriormente, las trayectorias con sus características requieren de ser entendidas y actualizadas en los marcos de encuadramiento definidos en las coyunturas económicas, políticas y socioculturales. Por factores económicos se entiende el modelo de desarrollo y su capacidad de dinamizar el mercado y producir empleos desde un proyecto nacional soberano; los factores políticos van más allá del régimen electoral e involucran la orientación gubernamental que refleja la correlación de fuerzas del bloque en el poder; y los factores socioculturales tienen que ver con los valores y prácticas que actualizan las relaciones de poder y de dominación o las disputas simbólicas por rupturas y superaciones.

Considerando la trayectoria reciente de construcción de un patrón de protección social inclusivo, amerita recordar que, desde fines de la década de 1970, aún bajo la dictadura militar, la sociedad brasileña construye una agenda política democrática, con la movilización intensa de movimientos sociales y partidos políticos. Más allá de la democracia competitiva electoral se propone una democracia social, con la universalización de los derechos sociales, en una de las sociedades más desiguales del mundo. El hecho de que la reforma de la protección social haya sido elaborada por los movimientos sociales que la plasmaron en la Constitución CF/88 es una de las características distintivas del sistema brasileño. Eso fue posibilitado por la fuerza de la coalición reformadora, por un lado, y por el proceso de la Asamblea Nacional Constituyente que ha permitido la fuerte participación popular. Además, los sectores conservadores, fuertemente atados a las prebendas del aparato estatal de la dictadura no fueron capaces de construir un proyecto hegemónico frente a una sociedad que demandaba un fuerte cambio.

El título más innovador de la CF/88 es el de "Orden social" en el cual se incluyen los derechos sociales, ambientales, culturales, de protección de grupos especiales, de normalización relativa a la ciencia y tecnología, a medios de comunicación y a los deportes. Son nuevos derechos y normas que no estuvieron presentes en las constituciones anteriores y que expresaban las demandas por la extensión de la ciudadanía y las necesidades de regulación estatal para promover el desarrollo y el bienestar social. Al interior de ese capítulo se construye un núcleo de la protección social bajo el título de "Seguridad social", que incluye las pensiones y jubilaciones, la salud y la asistencia social, con dotaciones específicas que conforman el presupuesto de la seguridad social. Aunque pensiones y salud fueron ya incluidas en las constituciones anteriores, estuvieron siempre limitadas a los trabajadores del mercado formal. Los principios que orientan la seguridad social están orientados a la universalización de la ciudadanía y el deber del Estado para garantizar los derechos sociales. Sin embargo, la naturaleza de los derechos es distinta cuando se trata de pensiones (derecho contractual), salud (derecho universal basado en la necesidad), asistencia (derecho basado en la insuficiencia de medios). Por supuesto, eso es motivo de tensiones y adaptaciones como, por ejemplo, las pensiones no-contributivas vistas como asistenciales y rechazadas constantemente por el sistema de pensiones. Por otro lado, los escasos recursos del presupuesto de la seguridad social fueron acaparados por las pensiones -un derecho contractual- en detrimento de los sistemas de 
salud y asistencia. Solamente después de algunas décadas, el sistema judicial pasó a incorporar los derechos sociales de la ciudadanía y permitir que su exigibilidad sea demandada judicialmente.

Una de las más importantes innovaciones fue la definición de salud y asistencia como derechos sociales a ser institucionalizados por medio de la construcción de sistemas descentralizados y participativos, dando lugar a la creación del Sistema Único de Salud (SUS) y el Sistema Único de Asistencia Social (SUAS). Esa arquitectura institucional involucró la construcción de redes con la participación de los tres niveles de gobierno - federal, estadual y municipal- con instancias de negociación capaces de generar pactos intergestores que refortalecieron al federalismo. Asimismo, se crearon instancias de participación social, como los consejos con funciones de control social y las conferencias en cada uno de los niveles gubernamentales, para la discusión y formación de políticas ${ }^{(27)}$.

Si bien hubo enormes avances en la construcción de la protección social, en términos legales e institucionales, también hubo trabas políticas y obstáculos financieros. La CF/88 definió como piso de los beneficios el sueldo mínimo, lo que elevó inmediatamente muchos de los beneficios asistenciales. También se expandió el gasto social con la expansión de la cobertura. Hubo preocupación para diversificar las fuentes de financiación de la seguridad social, evitando el carácter procíclico de las contribuciones sobre la nómina salarial y fueron creadas contribuciones sobre el lucro. Pero no hubo un pacto interclases capaz de asegurar los recursos necesarios para la protección social, con mayores aportes fiscales. El sistema tributario continuó siendo altamente regresivo y las medidas de combate a la inflación del Plan Real en 1994 inauguraron un mecanismo de desvinculación de los recursos del presupuesto de la seguridad social. O sea, en vez del presupuesto fiscal abastecer el de la seguridad social, lo que ocurrió fue un drenaje de recursos de la seguridad para solventar el déficit fiscal, aumentado por los intereses de la creciente deuda pública.
Durante los años del gobierno de Fernando Henrique Cardoso, no hubo fortalecimiento de la seguridad social, solo indirectamente, con el aumento del sueldo mínimo. En el área asistencial el gobierno buscó establecer relaciones con organizaciones no gubernamentales para la provisión de los servicios, en detrimento de la estructura pública del SUAS, en la cual la sociedad participaba como control social y no como proveedora de servicios. La opción de contratar proveedores privados se expandió legalmente hacia el área de salud. Sin embargo, los movimientos de la sociedad civil han continuado ejerciendo una fuerte influencia en el SUS, resistiendo a presiones privatistas incentivadas por el Fondo Monetario Internacional y el Banco Mundial, y avanzando en la construcción de un ejemplar programa de prevención del VIH-sida. La reforma de las pensiones alcanzó a establecer un techo y aumentar la edad para acceder a los beneficios en el sistema privado de pensiones, pero no tuvo éxito en el sistema público. $\mathrm{Si}$, por un lado, con el aumento del sueldo mínimo se ampliaba la distribución, por otro, al empujar a los trabajadores hacia el mercado de seguros complementarios se concentraban recursos en manos del capital financiero.

Los gobiernos del Partido de los Trabajadores (PT), con Luiz Inácio "Lula" da Silva, se beneficiaron del boom de commodities, lo que les ha permitido avanzar con medidas distributivas como el aumento del sueldo mínimo, las políticas de inclusión por cupos en las universidades, expansión de la red pública educacional y de las matrículas, y, el énfasis en el combate a la pobreza con la transferencia condicionada de ingresos por medio del programa Bolsa Familia. Se implementaron otros programas de apoyo a la población más empobrecida, pero no fueron alteradas ni la matriz productiva ni la estructura tributaria con las que podrían revertir el proceso de desindustrialización y de aumento de la informalidad, que se profundizó en el gobierno de Dilma Rousseff ${ }^{(28)}$. El sistema de salud se vio de alguna forma beneficiado con mayores dotaciones presupuestarias, aunque siguió subfinanciado, comparativamente 
con la dotación per cápita de países vecinos, como Argentina. El SUAS se fortaleció y alcanzó a desarrollar una institucionalidad y una profesionalización importante, además de la capilaridad de la red de servicios en el territorio nacional. El expresidente Lula hizo una reforma paramétrica en el sistema de pensiones del sector público, acercándolo al sistema privado ya reformado, pero para ser implementada en quienes ingresen al servicio público en los próximos años. Esa reforma mantuvo la tendencia de desplazar los mayores sueldos a los seguros privados.

El énfasis del gobierno de Lula en el combate a la pobreza resultó en programas de transferencia de ingresos y de promoción social, pero siempre desde una perspectiva individual, de permitir el acceso del sector más pobre al consumo. Se acuñó el eslogan de ascenso de los pobres a las capas medias, invirtiendo la pirámide de estratificación social. Sin embargo, a los nuevos consumidores no se les regaló derechos ciudadanos ni sustentabilidad del proyecto personal o político. La ideología meritocrática individualista los convirtió en sostenedores de la oposición conservadora en las elecciones presidenciales ${ }^{(29)}$.

Las grandes manifestaciones callejeras de 2013 pudieron expresar un rol muy diversificado de insatisfacciones de los jóvenes con la ausencia de perspectivas, denuncias de corrupción, falta de transparencia en el proceso decisorio acerca de la asignación de recursos para mega eventos como las Olimpiadas y la Copa Mundial de Fútbol. La crisis financiera se desbordó en crisis política y de legitimidad de un gobierno errático, seguida por el proceso de destitución de la presidenta.

El golpe mediático-jurídico-parlamentario que destituyó a la presidenta Dilma Rousseff repone el poder en manos de una coalición de conservadores, con fuerte representación en el Parlamento ${ }^{(28)}$. En el corto período de su gobierno, el expresidente Temer estableció un techo fijo para los gastos públicos por 20 años, congelando gastos sociales por debajo de lo necesario. También propuso dos reformas que apuntaban a la destrucción de los fundamentos de los derechos de los trabajados adquiridos en la década de 1930, en la era Vargas: la reforma de los derechos laborales y sindicales y la reforma de pensiones. La pérdida de derechos laborales se había iniciado ya en el gobierno de Cardoso, a título de flexibilización y adecuación de la legislación a una economía globalizada y competitiva. Si bien fueron creados contratos temporarios, las centrales sindicales tuvieron capacidad de resistir pérdidas más significativas en aquel entonces. Pero, ahora, con un gobierno netamente conservador apoyado por el Parlamento y por toda la burguesía, los cambios fueron más radicales, y alcanzaron la capacidad financiera de los sindicatos que ya venían perdiendo afiliados frente al proceso de transformación productiva. De esa forma, la pérdida de derechos laborales no pudo ser aminorada por las centrales sindicales, que perdieron mucha capacidad de movilización, y la propuesta de reforma del sistema de pensiones no tuvo éxito en función de que el presidente estaba denunciado por corrupción.

En las elecciones de 2018, hubo una fuerte participación del poder judicial -con la operación "Lava-Jato" (lavadero de autos)para manipular a la opinión pública e impedir la postulación de Lula como candidato favorito. Al resultar electo Jair Bolsonaro, -un parlamentario que se caracterizaba por su incompetencia, aliada a un discurso de defensa de la dictadura, de la tortura y del machismo- se forma una coalición liberal ultraconservadora, con apoyo de la alta burguesía financiera, de los militares y policías, iglesias evangélicas, y grupos de ultraderecha organizados en las redes sociales. A partir de entonces comienza un proceso de profundización del desmantelamiento de las políticas de protección social en un contexto de devastación de otras políticas públicas en áreas tan distintas como medio ambiente, reservas indígenas, relaciones exteriores, educación, comportamentales, etc.

\section{El andamiaje de la protección social}

El proceso histórico nos permite identificar algunos de los factores que explican el rápido derrumbe de la protección social que se 
produjo en apenas dos años de gobierno con la reforma de las pensiones, la militarización del Ministerio de Salud, la confrontación federativa en el enfrentamiento de la pandemia, la desestructuración de la red de asistencia social y el desmantelamiento de los sistemas de información de salud y asistencia.

El armado del andamiaje de la protección social se dio en dos momentos distintos: en 1930 y en 1988. En el gobierno de Vargas de 1930, el modelo corporativo incluyó a los trabajadores formales en la propia estructura del Estado, cogestionando el fragmentado sistema de pensiones y la estructura sindical autorizada por el gobierno. Los beneficios de la legislación laboral solo se aplicaban a trabajadores formales y los beneficios del sistema de pensiones estaban diferenciados y se negociaban con el gobierno, de acuerdo con la capacidad de presión de cada fracción de los trabajadores. Así, quedaron sin cobertura la gran masa de trabajadores autónomos, informales, domésticos y rurales. Por medio de la política social se reproducen las desigualdades del mercado y las injusticias sociales, además de actuar como potente factor de competición intraclase. La cooptación de los trabajadores como instrumento de legitimación del gobierno los convirtió en socios del pacto de poder cuando los beneficios se transformaron en privilegios y símbolos de estatus. La CF/88 realizó cambios en el sentido de reducir el control estatal sobre los sindicatos y la inclusión de pensiones no contributivas, pero no alteró sustancialmente los pilares de esas políticas.

En los países centrales, la consolidación del welfare state representó la expansión de la cobertura y la reducción de diferenciaciones entre los beneficiarios, factores apuntados como generadores de una masa de electores y técnicos que defienden la preservación de tales sistemas. Así, como en la fase de construcción del sistema los políticos se benefician de los créditos por la creación de beneficios concretos, en la fase del retroceso (retrenchment) las pérdidas son concretas y los beneficios difusos $^{(10)}$. Luego, el costo de las propuestas de cambios estructurales suele ser muy alto para los políticos, lo que hace que la resistencia generalizada y la defensa de los derechos sociales puedan impedir transformaciones que vayan más allá de los ajustes paramétricos.

El análisis de la trayectoria del caso brasileño evidencia la cristalización de los beneficios laborales y las pensiones como privilegios de grupos corporativos. Con la mitad de los trabajadores excluidos de los beneficios laborales, por su trabajo informal, los beneficios se inscribieron fuertemente en el imaginario colectivo como privilegios. En tal situación no es difícil tomar a los trabajadores formales o a los funcionarios públicos como chivos expiatorios de una grave crisis social que amenaza los hogares más pobres y a los cuales se imputa la falta de perspectiva para la juventud que va a pagar por tales privilegios. Privilegios corporativos, falta de perspectiva de la juventud y grave crisis económica son los ingredientes necesarios para que un gobierno liberal y un Parlamento ultraconservador tengan aprobado una reforma laboral que perjudica a los trabajadores y aumenta la informalidad.

Las reformas de los diferentes regímenes de pensiones públicos y privados fueron justificadas con base en la transición demográfica, el impacto sobre el déficit fiscal y la iniquidad de ese gasto público. Por otro lado, se justifica que quienes tengan sueldos más altos busquen complementar su pensión en seguros privados con base en la necesidad de aumentar el ahorro interno. Supone que eso permite inversiones en infraestructura y otros proyectos de largo plazo, como una palanca para el desarrollo nacional. Los mecanismos utilizados fueron el aumento de los requisitos para el acceso a las jubilaciones y pensiones y el establecimiento de un techo bastante bajo para las pensiones, de tal manera que funciona como estímulo para la búsqueda de una pensión complementaria en el mercado financiero. Los estudios han mostrado que tales promesas no se han cumplido. El proceso de acumulación del ahorro de los trabajadores en seguros privados y fondos de pensiones ha sido mayormente aplicado en títulos de la deuda pública, operaciones de corto plazo sin riesgos, que favorecen la 
manutención de altas tasas de interés, aumentando, por ende, el déficit público ${ }^{(30)}$.

La reforma del sistema de pensiones de 2019 fue todavía más radical. La propuesta enviada al Congreso preveía un cambio estructural, con la introducción del sistema de capitalización en cuentas individuales, a semejanza del sistema chileno que ya se encontraba en plena crisis con el empobrecimiento de los jubilados. Aunque el Congreso rechazó la propuesta de capitalización y de reducción de los beneficios a los trabajadores rurales y las pensiones asistenciales, se aprobaron medidas que disminuían los valores de las pensiones y aumentaban las restricciones al acceso a tales beneficios. Para los próximos 10 años, se prevé una reducción significativa del gasto público en pensiones, proveniente de cerca del $80 \%$ de los contribuyentes que ganan hasta dos sueldos mínimos. Las iniquidades se profundizarán, dado que las mujeres y la población más pobres, que alternan la informalidad con inserción en el mercado formal, tendrán más dificultad para cumplir los duros requisitos establecidos para el acceso, de los que fueron eximidos los militares.

Para una gran parte de los trabajadores que están en el mercado informal o fluctúan entre el formal y el informal, esas cuestiones les son ajenas, dado que los sindicatos han perdido gran parte de sus capacidades de movilización y la comunicación en defensa de la reforma los identificaba como los privilegiados.

\section{La propuesta de universalización}

En el caso del sistema de salud, la universalización propuesta en la CF/88 fue una efectiva transformación del modelo anterior, que transformó al sistema único en proveedor del derecho a una salud integral.

La implementación del SUS es concomitante a los intentos de su restricción y desmantelamiento. Los principales instrumentos de boicots fueron el subfinanciamiento de la atención a la salud, la baja inversión en la calificación del personal y en el desarrollo de ciencia y técnica, y la prioridad para la compra de servicios privados. La estrategia de defensa del SUS apostó al fortalecimiento institucional, la movilización de los actores políticos de base y de los gestores, parlamentarios e intelectuales, buscando establecer pisos mínimos de contribuciones de los entes federativos, ampliación de la atención primaria y de la cobertura. A diferencia del welfare state de los países centrales, en los que luego de su la creación prosigue la expansión, maduración e intentos de restricción y reformas, en nuestro caso se observa un permanente movimiento de idas y venidas, en función de los embates entre, por un lado, el conjunto de agencias que difunden el paradigma neoliberal y lo imponen por medio de los financiamientos a los estados endeudados y sus aliados nacionales y, por otro lado, las fuerzas que defienden el proyecto constitucional, una alianza que cambia a lo largo del proceso desde el movimiento de salubristas a los gestores del SUS.

En ese embate el principal mecanismo del gobierno para frenar la expansión y consolidación del SUS ha sido el déficit de financiación, justificado por la narrativa de que el principal problema siempre fue la gestión. Sin inversión en la gestión o en el combate a la corrupción, utilizando las principales agencias del sistema de salud como moneda de cambio con los partidos de apoyo al gobierno, se perpetúan los problemas de ineficiencia que justifican el subfinanciamiento, una rueda que se cierra en sí misma.

La consecuencia es el deterioro de los servicios públicos de excelencia que antes atendían a una parcela mínima de los trabajadores, pero que tiene peso en la opinión pública. Las dificultades de acceso se tornan una rutina que niega la seguridad implícita en el concepto del derecho a la salud, restaurando la condición de subalternidad de los usuarios en vez de su empoderamiento como ciudadanos, un verdadero contraderecho ${ }^{(31)}$.

A diferencia de los países centrales donde la construcción de los sistemas públicos universales de salud, como el National Health Service (NHS) de Inglaterra, se dieron con antelación a la capitalización de los servicios 
de salud, en el caso de Brasil, aún durante la dictadura, la política pública ha privilegiado la contratación hacia afuera, generando artificialmente, por medio de subsidios, un sector privado de proveedores de servicios de salud, articulados a un mercado monopólico del Estado. Situación que se mantuvo durante todo el tiempo de construcción del SUS.

Tres décadas después, con la ausencia de medidas de regulación en la constitución del SUS y con el fuerte empuje de la internacionalización del mercado de aseguradoras y proveedores de servicios de salud, la correlación de fuerzas entre los defensores del sistema público y los intereses privados se tornó un campo abierto de disputa por los fondos públicos.

La articulación entre los seguros privados de salud y los medios de comunicación se consolidó como socios o como marketing, de tal forma que los medios estuvieron, en todo momento, como los principales adversarios del SUS, hasta la pandemia, donde el SUS fue el único recurso disponible para toda la sociedad. La dinámica de intensa internacionalización y de conglomeración en los seguros y servicios privados fortaleció su lobby, fortaleciendo campañas políticas de parlamentarios y ejecutivos. Su proyecto de expansión se centra en la creación de un sistema que englobe los servicios públicos y privados, y en aumentar la obtención de recursos públicos en un mercado limitado por la exigüidad de clientes con capacidad contributiva.

Hay un aspecto cultural que amerita especial atención. El SUS es la única política pública totalmente fundada en el principio de la igualdad, en la que todos los ciudadanos, de acuerdo con sus necesidades, tienen derecho a la integralidad de los servicios de salud. En una sociedad enraizada en la esclavitud, la desigualdad es el principal principio organizador de las relaciones económicas y sociales, infiltrándose al interior de un Estado moderno que propone la ciudadanía universal. En consecuencia, se observa un doble movimiento: por un lado, el impulso de evadir un proyecto que iguala a los desiguales, como hizo la clase media, incluso con subsidios públicos, para poder mantener el seguro privado; por otro lado, la utilización de la red de relaciones de poder para tener un acceso diferencial a los servicios públicos en casos no cubiertos por los planes de salud, por ser enfermedades crónicas o trasplantes que no generan ganancias. El rechazo a ser un igual es constitutivo de la cultura brasileña y tener un plan privado de salud se ha transformado en un símbolo de distinción social.

La pandemia coloca al SUS en el centro de la crisis, aumentando su visibilidad y permitiendo que la sociedad entienda la complejidad del sistema, que involucra mucho más que los servicios de atención a la salud, como vigilancia sanitaria y epidemiológica, desarrollo científico y tecnológico, producción de insumos, fármacos y vacunas, sistemas de información, etc. La posición negacionista del presidente con relación a la gravedad de la pandemia, la obstrucción a las medidas de aislamiento y prevención propugnadas por gobiernos regionales y locales, su reproche a la vacunación y sus actitudes irresponsables en aglomeraciones públicas han generado innumerables consecuencias sanitarias y políticas. El desmantelamiento del sistema federal-cooperativo y su reemplazo por un sistema de confrontación tuvo como consecuencia el aumento aterrador del número de muertos, la deslegitimación de la autoridad sanitaria y el desmantelamiento del sistema nacional de informaciones en salud, la ausencia de planificación para compras de vacunas, entre otras medidas. La reacción vino por parte de los gobernadores, un federalismo de confrontación.

\section{El Sistema Único de Asistencia Social}

La creación del SUAS ha sido bastante obstaculizada por el gobierno que se rehusaba a aceptar la legislación infraconstitucional, imprescindible para la puesta en marcha del sistema. Sin contar con la misma movilización que el SUS, la asistencia tenía una trayectoria de acciones voluntarias, programas fragmentados y discontinuidad de acciones capitaneadas por las primeras damas y los religiosos. Sin embargo, en la transición democrática ya 
empezaba la discusión de la asistencia como derecho entre los liderazgos de las asociaciones profesionales del área. En la CF/88 mimetizaron el diseño del área de salud para su sistema de asistencia, pero alcanzaron a introducir un ingreso mínimo para ancianos y discapacitados en extrema pobreza. Aunque con condiciones de acceso muy restrictivas, el valor del beneficio fue equiparado a un salario mínimo en el texto constitucional. Por ende, aprobó la Ley Orgánica de Asistencia Social (LOAS) dando inicio al SUAS, empezando la institucionalización de un sistema público descentralizado y participativo.

La opción por medidas de combate a la pobreza en la década de 1990 creó una institucionalidad paralela al SUAS, directamente desde la primera dama hacia un conjunto de instituciones privadas, organizaciones no gubernamentales y proveedoras de servicios asistenciales. Mientras la institucionalidad del SUAS se vinculaba a las municipalidades y al sistema de pensiones, el programa Comunidad Solidaria corría en paralelo, alegando que las prácticas políticas de los gobiernos locales eran clientelares. Esa situación llevó a la paradoja de tener, en un determinado momento, dos ministerios para un área como la asistencial, tan desprovista de recursos.

La superación de tal fractura no se ha completado integralmente, pero avanzó hacia la unificación de los programas asistenciales, el fortalecimiento institucionalidad, la normalización de protocolos de atención y la ampliación de la cobertura, los beneficios y la capilaridad de la red de atención. El principal pilar de sustento del sistema, que fue mejorado durante las últimas décadas, es el registro único (CAD-Único), con información esencial sobre las necesidades y condiciones de vida de los beneficiarios, para permitir la concesión de beneficios, la planificación de las acciones, la toma de decisiones y, fundamentalmente, una acogida integral de las necesidades de los usuarios y familiares.

Durante la pandemia se aprobó una ayuda de emergencia para la población más empobrecida, cuya concesión fue implementada por un banco público, sin relación con el SUAS y sin utilizar el registro único.
Se provocaron humillantes filas de personas pobres en búsqueda del beneficio, en plena pandemia. Por falta de control, muchos de quienes alcanzaron a recibir tal beneficio no eran parte del grupo de los más necesitados. Por otro lado, el país descubrió lo que pasaron a llamarse "Ios invisibles", para designar la creciente miseria, pobreza e informalidad que aumentó con la pandemia.

Las formas tradicionales de boicots al derecho a la asistencia han sido el subfinanciamiento, la baja inversión en capacitación, gestión y mantenimiento de la estructura e incorporación tecnológica en la red de servicios. La prioridad política por aquellos programas asistenciales que no aseguren derechos de ciudadanía, permite que las demandas sean contenidas y los valores del beneficio se desactualicen. El gobierno Bolsonaro ha propuesto en diversas oportunidades y en la reforma del sistema de pensiones desvincular el Beneficio de Prestación Continua (BPC) del sueldo mínimo, para reducir el valor del beneficio, lo que ha sido rechazado por el Congreso.

La nueva propuesta del gobierno es alterar la dinámica del registro CAD-Único para que deje de ser llenado en la red descentralizada de cada municipalidad por el profesional que atiende las demandas y las deriva a los servicios, y pase a ser hecho por cada individuo, a través de Internet, en un banco de datos centralizado. Además de la exigencia del acceso y utilización de Internet, deja afuera al poder municipal y a la red de servicios del SUAS, cambiando también la lógica del acogimiento de las necesidades integrales y la derivación a los servicios públicos existentes en el territorio, para una demanda puntual en un proceso impersonal. Además, existe el riesgo de que ese banco de datos pueda ser vendido o cedido a terceros.

La baja capacidad de organización y vocalización de la población más pobre suele explicar el avance de los planteos del gobierno en dirección al desmantelamiento del SUAS y en la búsqueda de la de-constitucionalización de la asistencia como derecho social. Sin embargo, la existencia de una red de servicios en las municipalidades, el número 
de profesionales involucrados, los centros de formación existentes han formado una masa crítica que busca barrer el avance de tales propuestas y encuentra eco en el Congreso, en especial, cuando la pobreza y la miseria están avanzando velozmente.

Las políticas extremadamente conservadoras fortalecen y difunden valores machistas, misóginos, racistas, y así son contempladas las bases conservadoras y religiosas que apoyan al gobierno. Pero hay que considerar los factores culturales que avalan las propuestas gubernamentales en la medida en que parte de la sociedad continúa mirando a la población empobrecida con prejuicio, como vagabundos que no han buscado mejorar sus condiciones de vida, que son promiscuos, incapaces de planificar su familia y cuentan con los beneficios sociales para sobrevivir en vez de buscar su sustento por medio del trabajo.

Reubicar a la población más empobrecida en su debido lugar es una de las orientaciones centrales del bloque en el poder, lo que requiere restringir los derechos sociales y desmantelar la red de protección que ha sido armada.

\section{A MODO DE CIERRE}

Para finalizar, podemos señalar la incapacidad de las instituciones nacionales para impedir el derrumbe de la arquitectura democrática que se vino armando en las últimas décadas. El sistema de protección social brasileño buscó universalizar los derechos sociales de la ciudadanía por medio de la constitucionalización de tales derechos y de la institucionalización de sistemas nacionales descentralizados y participativos en áreas de salud y asistencia. Es indudable que se vencieron obstáculos financieros y políticos en idas y venidas, avances y retrocesos. Sin embargo, no fueron suficientes para enfrentar a un gobierno que representa las fuerzas más retrógradas de la sociedad aliada a las más dinámicas fracciones del capital. La trayectoria de la construcción de los sistemas de protección social apuntó las debilidades no superadas para la construcción de una democracia de ciudadanos.

Por el contrario, tenemos una alianza entre financistas, conservadores y autoritarios que busca revertir el estándar civilizatorio que se había alcanzado. Son los intereses de los sectores del capital financiero que se benefician de las políticas de reducción de la protección social a los trabajadores, obligados a buscar complementaciones en seguros privados; son los sectores más conservadores de la sociedad que apuntan al retroceso de los avances sociocomportamentales conquistados en las últimas décadas, representados mayormente por las iglesias neopentecostales; son los descendientes de la dictadura, como militares que añoran el autoritarismo represor y que hoy día son también coetáneos de otros defensores del autoritarismo represor como policías militares y milicianos.

Por otro lado, el sindicalismo ha perdido fuerza con las transformaciones en la producción y con las políticas de flexibilización y el retroceso de los derechos laborales y sindicales. De todas maneras, la fragmentación de la clase obrera entre trabajadores formales con derechos e informales excluidos casi nunca permitió la unificación de las luchas. La crisis económica asociada a una crisis política que reforma el pacto de poder no da espacio a la manutención de los derechos sociales y estrategias de inclusión social. Lo que se está diseñando es la reducción de derechos y la atribución de los costos de las transferencias a la población más pobre para que sean pagados por los trabajadores empleados. Como esa fórmula no se sostiene, lo que está en curso es una política pública de exterminio del excedente poblacional desechable, disfuncional al capitalismo actual(32), a través de una necropolítica ${ }^{(33)}$, o sea, el ejercicio del poder que implica la eliminación de una parte de la población. La alianza entre los socios del bloque de poder se justifica para imponer un orden de este tipo.

Sin embargo, las imposiciones de los intereses de la fracción del capital financiero deslegitiman a las autoridades, afectan los intereses de diferentes grupos y fracciones de clase de la burguesía y le impiden al 
capitalismo promover innovaciones y ampliar la productividad, como se probó en la pandemia con las restricciones comerciales de las patentes y la competición entre productores de vacunas. En las iniciativas de una ciudadanía insurgente en las favelas y en las periferias, comienza a conformarse un contramovimiento en la búsqueda de alternativas urbanas y productivas alrededor de lo común, pero que todavía no ha ganado fuerza para provocar transformaciones en el orden decadente del capitalismo actual ${ }^{(34,35)}$.

\section{REFERENCIAS BIBLIOGRÁFICAS}

1. Rimlinger G. Welfare policy and industrialization in Europe, America and Russia. New York: John Wiley and Sons Inc; 1971.

2. Wilensky $\mathrm{H}$. Industrial society and social welfare: The welfare state and equality: Structural and ideological roots of public expenditures. Berkeley: University of California Press; 1975.

3. Flora $\mathrm{P}$, Alber J. Modernization, democratization, and the development of welfare states in Western Europe. En: Flora P, Heidenheimer A. The development of the welfare states in Europe and America. New Brunswick: Transaction Press; 1981. p. 37-65.

4. Offe C. A Democracia partidária competitiva e o welfare state keynesiano: Fatores de estabilidade e desorganização. En: Offe C. Problemas estruturais do Estado capitalista. Rio de Janeiro: Biblioteca Tempo Universitário; 1984. p. 356-385.

5. Marshall TH. Cidadania, classe social e status. Rio de Janeiro: Zahar Editores; 1967.

6. Polanyi K. A grande transformação: As origens de nossa época. Rio de Janeiro: Editora Campus; 1980.

7. Esping-Andersen G. The three worlds of welfare capitalism. New Jersey: Princeton University Press; 1990.

8. Castel R. Les métamorphses de la question sociale. Paris: Gallimard; 1995.

9. Streeck W. The crisis of democratic capitalism. New Left Review. 2011;71:5-29.

10. Pierson P. The New Politics of the Welfare State. New York: Oxford University Press; 2001.

11. Mesa-Lago C. Social security in Latin America: pressure groups, stratification, and inequality. Pittsburgh: University of Pittsburgh; 1978.

12. Filgueira F. Welfare and democracy in Latin America: The development, crises, and aftermath of universal, dual and exclusionary social states. Geneva: United Nations Research Institute for Social Development for Social Development; 2005.

13. Steffen MW, Côrtes SV. Understanding social protection systems in Latin America and the Caribbean: Typologies and efforts of classification. Sociology Compass. 2018;12:e12634. doi.org/10.1111/soc4.12634.

14. Martínez-Franzoni J. Welfare regimes in Latin America: Capturing constellations of markets, families, and policies. Latin American Politics and Society. 2008;50(2):67-100. doi: 10.1111/j.1548-2456.2008.00013.x.

15. SeguranUbiergo A. The political economy of the welfare state in Latin America: Globalization, democracy, and development. Cambridge: Cambridge University Presss; 2007.

16. Gómez MU. From social insurance to poverty relief: Avatars of social protection in Latin America. En: Bada X, Rivera-Sánchez L. The Oxford Handbook of the Sociology of Latin America. New York: Oxford University Press; 2020. p. 1-18. doi: 10.1093/ oxfordhb/9780190926557.013.6C.

17. Gramsci A. Maquiavel, a política e o Estado moderno. Rio de Janeiro: Civilização Brasileira; 1968.

18. Fleury S. Estado sem cidadão: Seguridade social na América Latina. Rio de Janeiro: FIOCRUZ; 1994.

19. Fleury S. Estado sin ciudadanos. Buenos Aires: Lugar Editorial; 1997.

20. Fleury S. Dual, universal or plural? Health care models and issues in Latin America: Chile, Brazil and Colombia. En: Molina C, Nuñez J, (eds.). Health services in Latin America and Asia. Washington DC: Inter-American Development Bank; 2001. p. 3-36.

21. Fleury S, Belmartino S, Baris E. Reshaping health care systems in Latin America: A comparative analysis of Health Care Reforms in Argentina Brazil and Mexico. Ottawa: IDRC; 2000.

22. Fleury S. Building democracy in an emerging society: The challenge of the welfare state in Brazil. En: 
Nederveen-Pieterse J, Cardoso A. Brazil emerging: Inequality and emancipation. New York: Routledge; 2013. p. 11-31. doi: 10.4324/9780203482131.

23. Wood G, Gough I. Conclusion. Rethinking social policy in development contexts. En: Gough I, Wood G, et al. Insecurity and welfare regimes in Asia, Africa, and Latin America: Social policy in development countries. Cambridge: Cambridge University Press; 2008. p. 313326.

24. Honneth A. Luta por reconhecimento: A gramática moral dos conflitos sociais. São Paulo: Editora 34; 2003.

25. Fraser N. Social justice in the age of identity politics: Redistribution, recognition, and participation. En: Fraser N, Honneth A. Redistribution or recognition?: A political-philosophical exchange. London: Verso; 2003. p. 7-109.

26. Santos WG. Cidadania e justiça. Rio de Janeiro: Campus; 1979.

27. Fleury S. Brazil's health-care reform: social movements and civil society. The Lancet. 2011;377:17241725. doi: 10.1016/S0140-6736(11)60318-8.

28. Fleury S, Pinho CE. Liquefação da rede de proteção social no Brasil autoritário. Revista Katálysis. 2018;21(1). doi: 10.1590/1982-02592018v21n1p14.
29. Fleury S. The welfare state in Latin America: reform, innovation, and fatigue. Cadernos de Saúde Pública. 2017;33(2):e00058116. doi: 10.15090/0102-311X000 58116.

30. Pernasetti F. Miragens do amanhã: Previdência e rodadas de neoliberalização no Brasil pós 1988. [Tesis de Doctorado]. Rio de Janeiro: IPPUR/URFJ; 2021.

31. Fleury S, Bicudo V, Rangel G. Reacciones a la violencia institucional: estrategias de los pacientes frente al contra derecho a la salud en Brasil. Salud Colectiva. 2013;9(1):11-25. doi: 10.18294/sc.2013.197.

32. Nun J. Superpoblación relativa, ejercito industrial de reserva y masa marginal. Revista Latinoamericana de Sociología Buenos Aires. 1969;5(2):180-225.

33. Mbembe A. Necropolitica. São Paulo: Editora N-1; 2019.

34. Fleury S. La expansión de la ciudadanía. En: Pontificia Universidad Javeriana (org.). Inclusión social y nuevas ciudadanías: Condiciones para la convivencia y seguridad democrática. Bogotá: Pontificia Universidad Javeriana; 2003. p. 167-194.

35. Fleury S. Capitalismo, democracia, cidadania: contradições e insurgências. Revista Saúde em Debate. 2018;42(3):108-124. doi: 10.1590/0103-11042018S309.

FORMA DE CITAR

Fleury S. Derechos sociales: difíciles de construir, fáciles de destruir. Salud Colectiva. 2021;17:e3577. doi: 10.18294/ sc. 2021.3577.

Recibido: 30 abr 2021 | Versión final: 17 may 2021 | Aprobado: 1 jun 2021 | Publicado en línea: 7 jun 2021

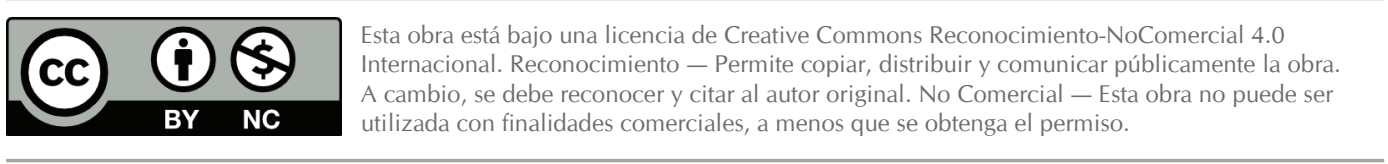

https://doi.org/10.18294/sc.2021.3577

Este artículo fue traducido del portugués por María Constanza Moretti 